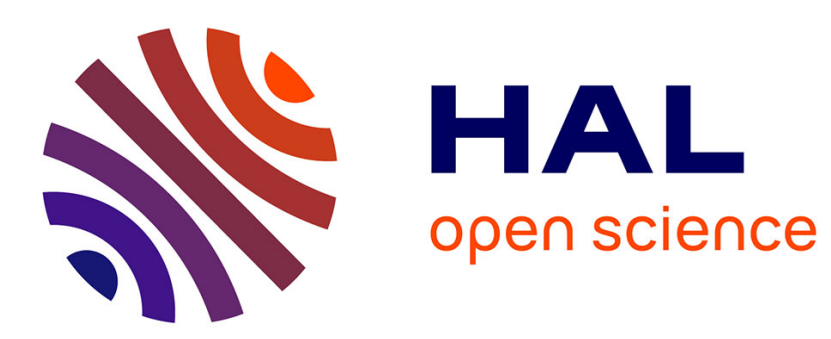

\title{
Discrete Cache Insertion Policies for Shared Last Level Cache Management on Large Multicores
}

\author{
Aswinkumar Sridharan, André Seznec
}

\section{To cite this version:}

Aswinkumar Sridharan, André Seznec. Discrete Cache Insertion Policies for Shared Last Level Cache Management on Large Multicores. [Research Report] RR-8816, INRIA-IRISA Rennes Bretagne Atlantique, équipe ALF. 2015. hal-01236706v2

\section{HAL Id: hal-01236706 https://hal.inria.fr/hal-01236706v2}

Submitted on 17 Dec 2015

HAL is a multi-disciplinary open access archive for the deposit and dissemination of scientific research documents, whether they are published or not. The documents may come from teaching and research institutions in France or abroad, or from public or private research centers.
L'archive ouverte pluridisciplinaire $\mathbf{H A L}$, est destinée au dépôt et à la diffusion de documents scientifiques de niveau recherche, publiés ou non, émanant des établissements d'enseignement et de recherche français ou étrangers, des laboratoires publics ou privés. 
Discrete Cache Insertion

Policies for Shared Last

Level Cache Management on Large Multicores

Aswinkumar Sridharan

André Seznec

RESEARCH

REPORT

$\mathbf{N}^{\circ} 8816$

December 2015

Project-Teams ALF 



\title{
Inzián
}

\section{Discrete Cache Insertion Policies for Shared Last Level Cache Management on Large Multicores}

\author{
Aswinkumar Sridharan \\ André Seznec \\ Project-Teams ALF \\ Research Report $\mathrm{n}^{\circ} 8816$ - December 2015 - 20 pages
}

\begin{abstract}
Multi-core processors employ shared Last Level Caches (LLC). This trend will continue in the future with large multi-core processors (16 cores and beyond) as well. At the same time, the associativity of this LLC tends to remain in the order of sixteen. Consequently, with large multicore processors, the number of cores that share the LLC becomes larger than the associativity of the cache itself. LLC management policies have been extensively studied for small scale multi-cores ( 4 to 8 cores) and associativity degree in the 16 range. However, the impact of LLC management on large multi-cores is essentially unknown, in particular when the associativity degree is smaller than the number of cores. In this study, we introduce Adaptive Discrete and deprioritized Application PrioriTization (ADAPT), an LLC management policy addressing the large multi-cores where the LLC associativity degree is smaller than the number of cores. ADAPT builds on the use of the Footprint-number metric. Footprint-number is defined as the number of unique accesses (block addresses) that an application generates to a cache set in an interval of time. We propose a monitoring mechanism that dynamically samples cache sets to estimate the Footprint-number of applications and classifies them into discrete (distinct and more than two) priority buckets. The cache replacement policy leverages this classification and assigns priorities to cache lines of applications during cache replacement operations. Footprint-number is computed periodically to account the dynamic changes in applications? behavior. We further find that deprioritizing certain applications during cache replacement is beneficial to the overall performance. We evaluate our proposal on 16, 20 and 24-core multi-programmed workloads and discuss other aspects in detail.
\end{abstract}

Key-words: Footprint-number, Discrete Priorities, More Cores than associativity 


\section{Discrete Cache Insertion Policies for Shared Last Level Cache Management on Large Multicores}

Résumé : Nous présentons ADAPT, une politique de remplacement pour les mémoires caches partagés de dernier niveau pour les processeurs multi-coeurs. ADAPT est plus efficace que les politiques de remplacement présentées auparavant.

Mots-clés : processeurs multicoeurs, mémoires cache. 


\section{Introduction}

In multi-core processors, the Last Level Cache (LLC) is usually shared by all the cores (threads) $)^{1}$. The effect of inter-thread interference due to sharing has been extensively studied in small scale multi-core contexts [4, 3, 12, 6, 7, 8, 1, 5, 9, 11, 2,. However, with advancement in process technology, processors are evolving towards packaging more cores on a chip. Future multi-core processors are still expected to share the last level cache among threads. Consequently, future multi-cores pose two new challenges. Firstly, the shared cache associativity is not expected to increase beyond around sixteen due to energy constraints, though there is an increase in the number of cores sharing the cache in multi-core processors. Hence, we are presented with the scenario of managing shared caches where (\#cores $\left.\geq \# l l c \_w a y s\right)$. Secondly, in large scale multi-core systems, the workload mix typically consists of applications with very diverse memory demands. For efficient cache management, the replacement policy must be aware of such diversity to enforce different priorities (more than two priorities) across applications. Moreover, there is an emerging trend of sharing the computing resources in the commercial grid systems where the basic computing structures are large multi-core processors with shared memory hierarchy. In such commercial systems, applications have different fairness and performance goals. Either the operating system or the hypervisor takes responsibility in accomplishing these goals. Therefore, the hardware must provide mechanisms to enforce software determined policies. Essentially, such requirement necessitates the replacement policy to enforce different priorities for applications $2^{2}$.

Prior studies 4, 3, 12, 1, 5, 2, have proposed novel approaches to predict the reuse behavior of cache lines and, hence their ability to utilize the cache. Typically, these approaches learn the application behavior as a consequence (hits or misses) of sharing the cache. Such an approach fairly reflects an application's ability to utilize the cache when the number of applications sharing the cache is small (2 or 4 ). However, when an application shares the cache with many co-running applications (typically, with diverse memory behaviors), its behavior at the shared cache may not necessarily reflect its ability to utilize the cache. Thus, using this approach leads to incorrect decisions on application priorities.

From the above discussions, it is clear that a cache replacement policy must satisfy the two requirements: A) allow enforcing discrete priorities across applications and B) efficiently capture an application behavior. Towards the combined goal, we introduce the metric Footprint-number to approximate dynamically the working-set size of applications at run-time. Footprint-number indicates the number of unique accesses (cache block addresses) that application generates to a cache set in an interval of time. Since Footprint-number explicitly approximates the working set size and quantifies the application behavior at run-time, it naturally provides scope for discretely (distinct and more than two priorities) prioritizing applications. We propose an insertion-priority-prediction algorithm that uses per application Footprint-number to guide the insertion priority of the cache lines of each application. Since Footprint-number is computed at run-time, dynamic changes in the application behavior are also captured. We further find that probabilistically de-prioritizing certain applications during cache insertions (that is, not inserting the cache lines) provides a scalable solution for efficient cache management.

Altogether, we propose Adaptive Discretized and De-prioritized Application PrioriTization $(A D A P T)$ for efficient management of large multi-core shared caches and make the following contributions:

-We consider cache replacement in shared caches in the context of (\#cores $\geq$ \#llc_ways): we find that observing the hit/miss pattern of applications to approximate their cache utility is not an efficient approach when the cache is shared by large number of applications, and a new mechanism is required.

-We propose a new metric Footprint-number to approximate application behavior at run-time

\footnotetext{
${ }^{1}$ Without loss of generality, we assume one thread/application per core.

${ }^{2}$ In this paper, we discuss only hardware based solution.
} 
and propose a prediction algorithm that uses Footprint-number to assign discrete (more than two) priorities for applications.

- From evaluation, we observe ADAPT to provide $4.7 \%$ improvement on the weighted speedup metric across 60 16-core multi-programmed workloads. We also show that ADAPT scales with respect to the number of applications that share the cache and also scales with larger cache sizes.

The remainder of the paper is organized as follows: In Section 2, we motivate the need for a new cache monitoring technique followed by detailing the proposed replacement algorithm in Section 3. We describe the experimental setup and evaluate our proposal in Sections 4 and 5 , respectively.

\section{Motivation}

We motivate the need for a new cache management technique as a consequence of (i) inefficient learning of application behavior in large-scale multi-cores. (ii) The complexity of other approaches that fairly isolate the application behavior.

Cache Management in large-scale multi-cores: A typical approach to approximate an application's behavior is to observe the hits and misses it encounters at the cache. Several prior mechanisms [3, 1, 12, 5, 2, have used this approach: the general goal being to assign cache space (not explicitly but by reuse prediction) to applications that could utilize the cache better. This approach works well when the number of applications sharing the cache is small (2 or 4 cores). However, such an approach becomes suboptimal when the cache is shared by large number of applications. We explain with set-dueling [4] as an example. Set-dueling: a randomly chosen pool of sets (Pool A for convenience) exclusively implements one particular insertion policy for the cache lines that miss on these sets. While another pool of sets (Pool B) exclusively implements a different insertion policy. A saturating counter records the misses incurred by either of the policies. In particular, misses on Pool $A$ increment, while the misses on Pool $B$ decrement the saturating counter, which is 10-bit in size. The switching threshold between the two policies is 512. They observe that choosing as few as 32 sets per policy is sufficient. TA-DRRIP[1] uses set-dueling to learn between SRRIP and BRRIP insertion policies. SRRIP handles mixed (recency-friendly pattern mixing with scan) and scan (long sequence of no reuse cache lines) type of access patterns, BRRIP handles thrashing patterns. Readers are requested to refer the original paper [1] for further details.

Under set-dueling, TA-DRRIP learns SRRIP policy for all applications, including the ones which have working-set larger than the cache also. However, applications with working-set size larger than the cache cause thrashing when they share the cache with other (cache-friendly) applications. Performance can be improved based on the intuition that applications with larger working-set size causes thrashing and therefore, explicitly preventing them from competing with the non-thrashing (or, cache-friendly) applications for the cache space. In other words, implementing BRRIP policy for these thrashing applications will be beneficial to the overall performance. Figure 1a confirms this premise. The bar labeled TA-DRRIP(forced) is the implementation where we force BRRIP policy on all the thrashing applications. Performance shown is normalized to TA-DRRIP. As we see from figure, the latter achieves speed-up close to 2.8 than the default implementation of TA-DRRIP. The experiments are performed on a 16MB, 16-way associative cache, which is shared by all sixteen applications. Table 3 shows other simulation parameters. Results in Figure 1 are averaged from all the 60 16-core workloads. Also, from bars 1 and 2, we see that the observed behavior of TA-DRRIP is not dependent on the number of sets dedicated for policy learning.

Figures $1 \mathrm{~b}$ and $1 \mathrm{c}$ show the MPKIs of individual applications when thrashing applications are forced to implement BRRIP insertion policy. For thrashing applications, there is little change in their MPKIs, except cactusADM. cactusADM suffers close to $40 \%$ increase in its MPKI and 


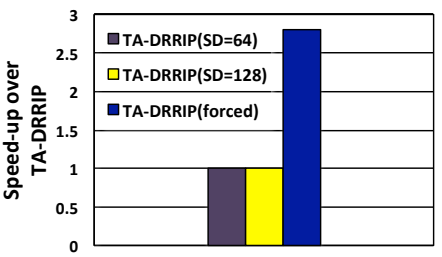

(a) Forced BRRIP priority

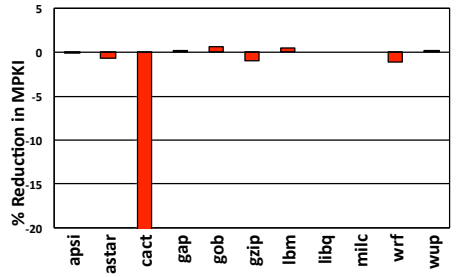

(b) MPKI for thrashing applications

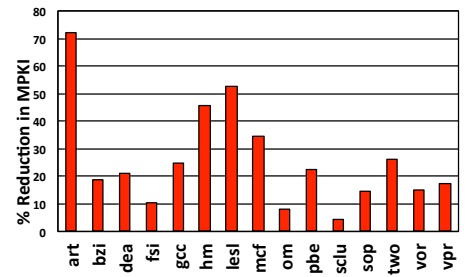

(c) MPKI reduction for others

Figure 1: Impact of implementing BRRIP policy for thrashing applications

$8 \%$ slow-down in its IPC while other thrashing applications show a very marginal change in their IPCs. However, non-thrashing applications show much improvement in their MPKIs and IPCs. For example, in Figure 1c, art saves up to $72 \%$ of its misses when thrashing applications implement BRRIP insertion policy as compared to when they also implement SRRIP insertion policy. Thus, thrashing applications implementing BRRIP as their insertion policy is beneficial to the overall performance. However, in practice, TA-DRRIP does not implement BRRIP for thrashing applications and loses out on the opportunity for performance improvement. Similarly, SHiP [5] which learns from the hits and misses of cache lines at the shared cache, suffers from the same problem. Thus, we infer that observing the hit/miss results of cache lines to approximate application behavior is not efficient in the context of large-scale multi-cores.

Complexity in other approaches: On the other hand, reuse-distance based techniques [33, 37, 36, 35] explicitly compute the reuse distance values of cache lines. However, they involve significant overhead due to storage and related bookkeeping operations. Further, these techniques are either dependent on the replacement policy [36, 37] or require modifying the cache tag arrays 33, 35. Similarly, cache partitioning techniques [30, 29, 10, 31] incur significant overhead due to larger associative (up to 128/256-way) tag structures, or require modification to the replacement policies to adapt to their needs 31] 30]. From these discussions, we see that a simple, efficient and scalable cache monitoring mechanism is required. Further, recall that cache replacement policy in large multi-core processors to be application-aware, and enforce different priorities (discrete: $>2$ ). Therefore, an efficient cache management technique must augment a cache monitoring mechanism that conforms to the two goals.

\section{Adaptive discretized and de-prioritized cache insertions (ADAPT)}

Adaptive and Discrete Application PrioriTization, ADAPT, consists of two components: (i) the monitoring mechanism and (ii) the insertion-priority algorithm. The first component monitors the cache accesses (block addresses) of each application and computes its Footprint-number, while the second component infers the insertion priority for the cache lines of an application using its Footprint-number.Firstly, we describe the design, operation and cost of the monitoring mechanism. Then, describe in detail the insertion-priority algorithm.

\subsection{Collecting Footprint-number}

Definition: Footprint-number of an application is the number of unique accesses (block addresses) that it generates to a cache set. However, during execution, an application may exhibit change in its behavior and hence, we define its Sliding Footprint-number ${ }^{3}$ as the number of

\footnotetext{
${ }^{3}$ However, we just use the term Footprint-number throughout.
} 

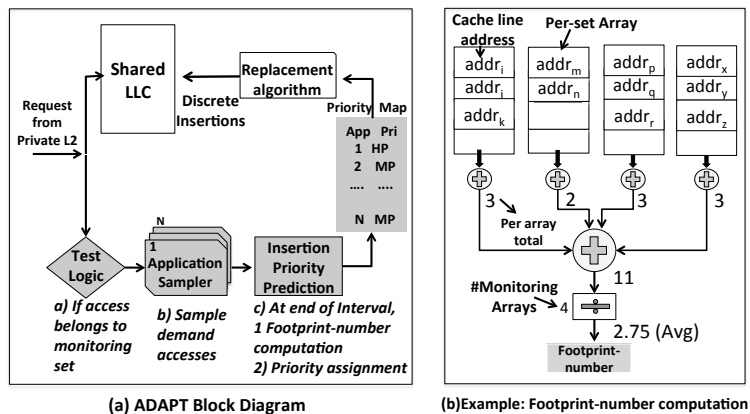

Figure 2: (a) ADAPT Block Diagram and b) example for Footprint-number computation

unique accesses it generates to a set in an interval of time. We define this interval in terms of the number of misses at the shared last level cache since only misses trigger new cache blocks to be allocated. However, sizing of this interval is critical since the combined misses of all the applications at the shared cache could influence their individual (sliding) Footprint-number values computed. A sufficiently large interval mitigates the impact of the total misses on the individual Footprint-number values. To fix the interval size, we perform experiments with $0.25 \mathrm{M}, 0.5 \mathrm{M}$, $1 \mathrm{M}, 2 \mathrm{M}$ and $4 \mathrm{M}$ interval sizes. Among, $0.25,0.5$ and $1 \mathrm{M}$ misses, $1 \mathrm{M}$ gives the best results. And, we do not observe any significant difference in performance between $1 \mathrm{M}$ and $4 \mathrm{M}$ interval sizes. Further, 1 Million misses on average correspond to $64 \mathrm{~K}$ misses per application and is roughly four times the total number of blocks in the cache, which is sufficiently large. Hence, we fix the interval size as $1 \mathrm{M}$ last level cache misses.

Another point to note is that Footprint-number can only be computed approximately because (i) cache accesses of an application are not uniformly distributed across cache sets. (ii) Tracking all cache sets is impractical. However, a prior study [6 has shown that the cache behavior of an application can be approximated by sampling a small number of cache sets (as few as 32 sets is enough). We use the same idea of sampling cache sets to approximate Footprint-number. From experiments, we observe that sampling 40 sets are sufficient.

Design and Operation: Figure $2 \mathrm{a}$ shows the block diagram of a cache implementing ADAPT replacement algorithm. In the figure, the blocks shaded with gray are the additional components required by ADAPT. The test logic checks if the access (block address) belongs to a monitored set and if it is a demand acces: 4 , and then it passes the access to the application sampler. The application sampler samples cache accesses (block addresses) directed to each monitored set. There is a storage structure and a saturating counter associated with each monitored set. The storage structure is essentially an array which operates like a typical tag-array of a cache set.

First, the cache block address is searched. If the access does not hit, it means that the cache block is a unique access. It is added into the array and the counter, which indicates the number of unique cache blocks accessed in that set, is incremented. On a hit, only the recency bits are set to 0. Any policy can be used to manage replacements. We use SRRIP policy. All these operations lie outside the critical path and are independent of the hit/miss activities on the main cache. Finally, it does not require any change to the cache tag array except changing the insertion priority.

Example: Figure $2 \mathrm{~b}$ shows an example of computing Footprint-number. For simplicity, let us assume we sample 4 cache sets and a single application. In the diagram, each array belongs to a separate monitored set. An entry in the array corresponds to the block address that accessed the set. We approximate Footprint-number by computing the average from all the sampled sets. In this example, the sum of all the entries from all the four arrays is 11. And, the average is

\footnotetext{
${ }^{4}$ Only demand accesses update the recency state
} 
2.75. This is the Footprint-number for the application. In a multi-core system, there are as many instances of this component as the number of applications in the system. Footprint-number for all applications is recomputed every 1 Million misses in the last level cache, as mentioned earlier.

Monitoring in a realistic system: In the paper, we assume that one thread per core. Therefore, we can use the core ID for the thread. On an SMT machine, the thread number/ID would have to be transmitted with the request from the core for our scheme to work properly.

If an application migrates (on a context-switch) to another core, the replacement policy applied for that application during the next interval will be incorrect. But, the interval is not long (1Million LLC misses). The correct Footprint-number and insertion policy will be reestablished in the following monitoring interval onward. In data-centers or server systems, tasks or applications are not expected to migrate often. A task migrates only in exceptional cases like shutdown or, any power/performance related optimization. In other words, applications execute(spend) sufficient time on a core for the heuristics to be implemented. Finally, in the study, like prior works [4, 3, 1, 2, 5, 6, we target systems in which LLC is organized as multiple banks with uniform access latency rather than systems with distributed last level caches.

\subsection{Footprint-number based Priority assignment}

Like prior studies [1, 5, 2, 39, we use 2 bits per cache line to represent re-reference prediction value (RRPV). RRPV '3' indicates the line will be reused in the distant future and hence, a cache line with RRPV of 3 is a candidate for eviction. On a cache hit, only the cache line that hits is promoted to RRPV 0, indicating that it will be reused immediately. On insertions, unlike prior studies, we explore the option of assigning different priorities (up to 4) for applications leveraging the Footprint-number metric.

We propose an insertion-priority-prediction algorithm that statically assigns priorities based on the Footprint-number values. The algorithm assumes that the LLC associativity is sixteen. However, it still works for larger associative caches as we show later. Table 1 summarizes the insertion priorities for each classification. Experiments are performed by varying the high-priority range between $[0,3]$ and $[0,8]$ (6 different ranges), keeping the low-priority range unaffected. Similarly, by keeping the high-priority range [0,3] constant, we change the low-priority range between $(7,16)$ to $(12,16)$ (6 different ranges). In total, from 36 different experiments we fix the priority-ranges. A dynamic approach that uses run-time information to assign priorities is more desirable. We defer this approach to future work. Priority assignments are as follows:

High Priority: All applications in the Footprint-number range [0,3] (both included) are assigned high-priority. When the cache lines of these applications miss, they are inserted with RRPV 0. Intuition:Applications in this category have working sets that fits perfectly within the cache. Typically, the cache lines of these applications have high number of reuses. Also, when they share the cache, they do not pose problems to the co-running applications. Hence, they are given high-priority. Inserting with priority 0 allows the cache lines of these applications to stay in the cache for longer periods of time before being evicted.

Medium Priority: All applications in the Footprint-number range (3,12] (3 excluded and 12 included) are assigned medium priority. Cache lines of the applications in this category are inserted with value 1 and rarely inserted with value 2. Intuition: Applications under this range of Footprint-number have working set larger than the high-priority category however, fit within the cache. From analysis, we observe that the cache lines of these applications generally have moderate reuse except few applications. To balance mixed reuse behavior, one out of the sixteenth insertion goes to low priority 2, while inserted with medium priority 1 , otherwise.

Low Priority: Applications in the Footprint-number range $(12,16)$ are assigned low priority. Cache lines of these applications are generally inserted with RRPV 2 and rarely with medium priority 1 ( 1 out of 16 cache lines). Intuition: Applications in this category typically have mixed access patterns : $\left(\{a 1, a 2\}^{k}\{s 1, s 2, s 3 . . s n\}^{d}\right)$ with $k$ and $d$ sufficiently small and $k$ slightly greater than $d$, as observed by TA-DRRIP[1]. Inserting the cache lines of these applications with low 
Table 1: Insertion Priority Summary

\begin{tabular}{|c|c|c|}
\hline Priority Leve & \multicolumn{2}{|c|}{ Insertion Value } \\
\hline High (HP) & \multicolumn{2}{|l|}{0} \\
\hline Medium (MP) & \multicolumn{2}{|c|}{1 but $1 / 16$ th insertion at LP } \\
\hline Low (LP) & \multicolumn{2}{|c|}{2 but $1 / 16$ th at MP } \\
\hline Least (LstP) & \multicolumn{2}{|c|}{ Bypass but insert $1 / 32$ nd at LP } \\
\hline \multicolumn{3}{|c|}{ Table 2: Cost on $16 \mathrm{MB}, 16$-way LLC } \\
\hline Policy & Storage cost & $\mathbf{N}=24$ cores \\
\hline TA-DRRIP & 16-bit/app & 48 Bytes \\
\hline EAF-RRIP & 8-bit/address & $256 \mathrm{~KB}$ \\
\hline SHiP & SHCT table\&PC & $65.875 \mathrm{~KB}$ \\
\hline ADAPT & 865 Bytes/app & $24 \mathrm{~KB}$ appx \\
\hline
\end{tabular}

priority 2 ensures (i) cache lines exhibiting low or no reuse at all get filtered out quickly and (ii) cache lines of these applications have higher probability of getting evicted than high and medium priority applications ${ }^{5}$

Least Priority: Applications with Footprint-number range $(>=16)$ are assigned least priority. Only one out of thirty-two accesses are installed at the last level cache with least priority 3. Otherwise, they are bypassed to the private Level 2. Intuition: Essentially, these are applications that either exactly fit in the cache (occupying all sixteen ways) or with working sets larger than the cache. These applications are typically memory-intensive and when run along with others cause thrashing in the cache. Hence, both these type of applications are candidates for least priority assignment. The intuition behind bypassing is that when the cache lines inserted with least priority are intended to be evicted very soon (potentially without reuse), bypassing these cache lines will allow the incumbent cache lines to better utilize the cache. Our experiments confirm this assumption. In fact, bypassing is not just beneficial to ADAPT. It can be used as a performance booster for other algorithms, as we show in the evaluation section.

\subsection{Hardware Overhead}

The additional hardware required by our algorithm is the application sampler and insertion priority prediction logic. The application sampler consists of an array and a counter. The size of the array is same as the associativity. From Section 3.2, recall that we assign the same priority(least) to applications that exactly fit in the cache as well as the thrashing applications because, on a 16-way associative cache, both classes of applications will occupy a minimum of 16 ways. Hence, tracking 16 (tag) addresses per set is sufficient. The search and insertion operations on the array are very similar to that of a cache set. The difference is that we don't store the entire tag bits. Only the most significant 10 bits are stored per cache block. Explanation: the probability of two different cache lines having all the 10 bits same is very low: $\left(1 / 2^{x}\right) /\left(2^{10} / 2^{x}\right)$, where $x$ is the number of tag bits. That is, $1 / 2^{10}$. Even so, there are separate arrays for each monitoring set. Plus, applications do not share the arrays. Hence, 10 bits are sufficient to store the tag address. 2 bits per entry are used for bookkeeping. Additionally, 8 bits are required for head and tail pointers ( 4 bits each) to manage search and insertions.

Storage overhead per set is 204 bits and we sample 40 sets. Totally, 204 bits $\times 40=8160$ bits. To represent an application's Footprint-number and priority, two more bytes (1 byte each) are needed. To support probabilistic insertions, three more counters each of size one byte are required. Therefore, storage requirement per application sampler is $[8160$ bits +40 bits $]=$ 8200bits/application. In other words, 1KB (appx) per application.

\footnotetext{
${ }^{5}$ It means that transition from 2 to 3 happens quicker than 0 to 3 or 1 to 3 thereby allowing HP and MP applications to stay longer in the cache than LP applications.
} 
Table 3: Baseline System Configuration

\begin{tabular}{|l|l|}
\hline Processor Model & $\begin{array}{l}\text { 4-way OoO, 128 entry ROB, 36 RS, 36- } \\
\text { 24 entries LD-ST queue }\end{array}$ \\
\hline Branch predictor & TAGE, 16-entry RAS \\
\hline IL1 \& DL1 & $\begin{array}{l}32 \mathrm{~KB} ; \text { LRU; next-line prefetch;I\$:2- } \\
\text { way;D } \$: 8-\text { way; 64 bytes line }\end{array}$ \\
\hline L2 (unified) & $\begin{array}{l}\text { 256KB,16-way, 64 bytes line, DRRIP, } \\
\text { 14-cycles, 32-entry MSHR and 32-entry } \\
\text { retire-at-24 WB buffer }\end{array}$ \\
\hline LLC (unified) & $\begin{array}{l}\text { 16MB, 16-way, 64 bytes line, TA- } \\
\text { DRRIP, 24 cycles, 256-entry MSHR } \\
\text { and 128-entry retire-at-96 WB buffer }\end{array}$ \\
\hline $\begin{array}{l}\text { Main-Memory } \\
\text { (DDR2) }\end{array}$ & $\begin{array}{l}\text { cycles, 8 } 8 \text { banks,4KB row, XOR- } \\
\text { mapped[28 }\end{array}$ \\
\hline
\end{tabular}

Table 2 compares the hardware cost of our technique with two other techniques. Though ADAPT requires more storage compared to TA-DRRIP [1, it provides higher performance improvement. ADAPT is also better compared to EAF [2] and SHiP [5] in both storage and performance aspect ${ }^{6}$ for the larger number of cores (larger than associativity) that we assume. It should be noted that ADAPT does not require dedicating some cache sets for policy learning. Regarding energy consumption, we empirically conjecture that the monitoring system will consume approximately $1 / 25^{\text {th }}$ of the power of the main cache tag array. (40 sets per application and 16 applications results in $1 / 25^{\text {th }}$ of accesses directed to the monitoring cache).

\section{Experimental Study}

\subsection{Methodology}

For our study, we use BADCO [19] cycle-accurate x86 CMP simulator. Table 3 shows our baseline system configuration. We do not enforce inclusion in our cache-hierarchy and all our caches are write-back caches. LLC is $16 \mathrm{MB}$ and organized into 4 banks. We model bank-conflicts, but with fixed latency for all banks like prior studies [1, 5, 2,.A VPC[7] based arbiter is used to schedule requests from L2 to LLC. We use memory model for our study like[2]: only row-hits and row-conflicts are modeled.

\subsection{Benchmarks}

We use benchmarks from SPEC 2000 and 2006 and PARSEC benchmark suites, totaling 36 benchmarks (31 from SPEC and 4 from PARSEC and 1 Stream benchmark). Table 4 shows the classification of all the benchmarks and Table 5 shows the empirical method used to classify memory intensity of a benchmark based on its Footprint-number and L2-MPKI when run alone on a 16MB, 16-way set-associative cache. In Table 4 the column Fpn(A) represents Footprintnumber value obtained by using all sets while the column $\mathrm{Fpn}(\mathrm{S})$ denotes Footprint-number computed by sampling. Only vpr shows $>1$ difference in Footprint-number values. Only to report the upper-bound on the Footprint-numbers, we use 32-entry storage. In our study, we use only 16-entry array.

We use a selective portion of 500M instructions from each benchmark. We fast-forward the first $200 \mathrm{M}$ instructions (warm-up all $\mathrm{h} / \mathrm{w}$ structures) and simulate the next $300 \mathrm{M}$ instructions. If an application finishes execution, it is re-executed from the beginning.

${ }^{6} \mathrm{HW}$ cost of EAF depends on the total number of cache lines. 
Table 4: Benchmark classification based on Footprint-number and L2-MPKI.

\begin{tabular}{|c|c|c|c|c|c|c|c|c|c|}
\hline Name & Fpn(A) & Fpn(S) & L2-MPKI & Type & Name & $\overline{F p n(A)}$ & Fpn(S) & L2-MPKI & Type \\
\hline black & 7 & 6.9 & 0.67 & VL & bzip & 4.15 & 4.03 & 25.25 & M \\
\hline calc & 1.33 & 1.44 & 0.05 & VL & gap & 23.12 & 23.35 & 1.28 & $\mathrm{M}$ \\
\hline craf & 2.2 & 2.4 & 0.61 & VL & gob & 16.8 & 16.2 & 1.28 & M \\
\hline deal & 2.48 & 2.93 & 0.5 & VL & $\mathrm{hmm}$ & 7.15 & 6.82 & 2.75 & M \\
\hline eon & 1.2 & 1.2 & 0.02 & VL & lesl & 6.7 & 6.3 & 20.92 & M \\
\hline fmine & 6.18 & 6.12 & 0.34 & VL & $\mathrm{mcf}$ & 11.9 & 12.4 & 24.9 & M \\
\hline h26 & 2.35 & 2.53 & 0.13 & VL & omn & 4.8 & 4 & 6.46 & M \\
\hline nam & 2.02 & 2.11 & 0.09 & VL & sopl & 10.6 & 11 & 6.17 & M \\
\hline sphnx & 5.2 & 5.4 & 0.35 & VL & twolf & 1.7 & 1.6 & 16.5 & M \\
\hline tont & 1.6 & 1.5 & 0.75 & VL & wup & 24.2 & 24.5 & 1.34 & M \\
\hline swapt & 1 & 1 & 0.06 & VL & apsi & 32 & 32 & 10.58 & $\mathrm{H}$ \\
\hline gcc & 3.4 & 3.2 & 1.34 & $\mathrm{~L}$ & astar & 32 & 32 & 4.44 & $\mathrm{H}$ \\
\hline mesa & 8.61 & 8.41 & 1.2 & $\mathrm{~L}$ & gzip & 32 & 32 & 8.18 & $\mathrm{H}$ \\
\hline pben & 11.2 & 10.8 & 2.34 & $\mathrm{~L}$ & libq & 29.7 & 29.6 & 15.11 & $\mathrm{H}$ \\
\hline vort & 8.4 & 8.6 & 1.45 & $\mathrm{~L}$ & milc & 31.42 & 30.98 & 22.31 & $\mathrm{H}$ \\
\hline vpr & 13.7 & 14.7 & 1.53 & $\mathrm{~L}$ & wrf & 32 & 32 & 6.6 & $\mathrm{H}$ \\
\hline fsim & 10.2 & 9.6 & 1.5 & $\mathrm{~L}$ & cact & 32 & 32 & 42.11 & $\mathrm{VH}$ \\
\hline sclust & 8.7 & 8.4 & 1.75 & $\mathrm{~L}$ & $\mathrm{lbm}$ & 32 & 32 & 48.46 & $\mathrm{VH}$ \\
\hline art & 3.39 & 2.31 & 26.67 & M & STRM & 32 & 32 & 26.18 & $\mathrm{VH}$ \\
\hline
\end{tabular}

Table 5: Empirical Classification Methodology

\begin{tabular}{|c|c|c|}
\hline FP-num & L2 MPKI & Memory Intensity \\
\hline \multirow{3}{*}{$<16$} & $<1$ & VeryLow (VL) \\
& {$[1,5)$} & Low (L) \\
& $>5$ & Medium (M) \\
\hline \multirow{3}{*}{$>16$} & $<5$ & Medium (M) \\
& {$[5,25)$} & High (H) \\
& $>25$ & VeryHigh (VH) \\
\hline
\end{tabular}

\subsection{Workload Design}

Table 6 summarizes our workloads. For 4 and 8-core workloads, we study with $4 \mathrm{MB}$ and $8 \mathrm{MB}$ shared caches while 16, 20 and 24-core workloads are studied with a $16 \mathrm{MB}$ cache since we target caches where \#applications $\geq$ \#llcassociativity.

Table 6: Workload Design

\begin{tabular}{|c|c|c|c|}
\hline Study & \#Workloads & Composition & \#Instructions \\
\hline 4-core & 120 & Min 1 thrashing & $1.2 \mathrm{~B}$ \\
8-core & 80 & Min 1 from each class & $2.4 \mathrm{~B}$ \\
16-core & 60 & Min 2 from each class & $4.8 \mathrm{~B}$ \\
20 \&24-core & 40 & Min 3 from each class & $6 \& 7.2 \mathrm{~B}$ \\
\hline
\end{tabular}

\section{Results and Analysis}

\subsection{Performance on 16-core workloads}

Figure 3 shows performance on the weighted-speedup metric over the baseline TA-DRRIP and three other state-of-the-art cache replacement algorithms. We evaluate two versions of ADAPT: one which inserts all cache lines of least priority applications (referred as $A D A P T$ ins) and the version which mostly bypasses the cache lines of least priority applications (referred as $A D A P T_{-}$bp32). Our best performing version is the one that bypasses the cache lines of thrashing applications. Throughout our discussion, we refer to ADAPT as the policy that implements bypassing. From Figure 3, we observe that ADAPT consistently outperforms other cache replacement policies. It achieves up to $7 \%$ improvement with $4.7 \%$ on average.

As mentioned in the motivation, under set-dueling, applications with working-set larger than the cache implement SRRIP policy, which causes higher contention and thrashing in the cache. 


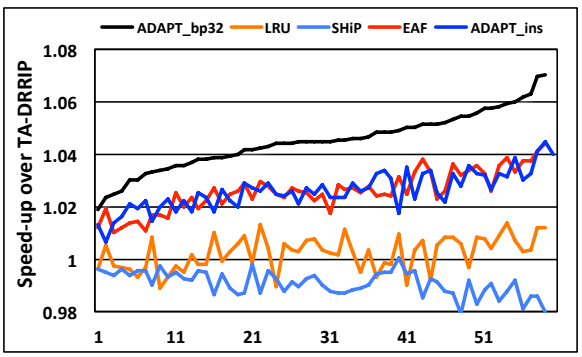

Figure 3: Performance of 16-core workloads

Similarly, SHiP learns the reuse behavior of region of cache lines (grouped by their PCs) depending on the hit/miss behavior. A counter records the hits (indicating near-immediate) and misses (indicating distant) reuse behavior for the region of cache lines. Since SHiP implements SRRIP, it observes similar hit/miss pattern as TA-DRRIP for thrashing applications. Consequently, like TA-DRRIP, it implements SRRIP for all applications. Only $3 \%$ of the misses are predicted to have distant reuse behavior. The marginal drop in performance (1.1\%appx) is due to inaccurate distant predictions on certain cache-friendly applications. Overall, TA-DRRIP and SHiP are not able to distinguish applications. On the other hand, ADAPT uses Footprint-number metric to efficiently distinguish across applications.

LRU inserts the cache lines of all applications at MRU position. However, cache-friendly applications only partially exploit such longer most-to-least transition time because the MRU insertions of thrashing applications pollute the cache. On the other hand, ADAPT efficiently distinguishes applications. It assigns least priority to thrashing applications and effectively filter out their cache lines, while inserting recency-friendly applications with higher priorities, thus achieving higher performance.

The EAF algorithm filters recently evicted cache addresses. On a cache miss, if the missing cache line is present in the filter, the cache line is inserted with near-immediate reuse (RRPV 2). Otherwise, it is inserted with distant reuse (RRPV 3). In EAF, the size of the filter is such that it is able to track as many misses as the number of blocks in the cache (that is, working-set twice the cache). Hence, any cache line that is inadvertently evicted from the cache falls in this filter and gets near-immediate reuse prediction. Thus, EAF achieves higher performance compared to TADRRIP, LRU and SHiP. Interestingly, EAF achieves performance comparable to ADAPT_ins. On certain workloads, it achieves higher performance while on certain workloads it achieves lesser performance. This is because, with ADAPT (in general), applications with smaller Footprintnumber are inserted with RRPV 0 or 1 . But, when such applications have poor reuse, EAF (which inserts with RRPV 2 for such applications) filters out those cache lines. On the contrary, applications with smaller Footprint-number but moderate or more number of reuses, gain from ADAPT's discrete insertions. Nevertheless, ADAPT (with bypassing) consistently outperforms EAF algorithm. We observe that the presence of thrashing applications causes the filter to get full frequently. As a result EAF is only able to partially track the application's (cache lines). On the one hand, some cache lines of non thrashing (recency-friendly) that spill out of the filter get assigned a distant (RRPV 3). On the other hand, cache lines of the thrashing applications that occupy filter positions get near-immediate (RRPV 2) assignment.

\subsection{Impact on Individual Application Performance}

We discuss the impact of ADAPT on individual application's performance. The results are averaged from all the sixty 16-core workloads. From Figures 3, 4 \& 5, we observe that bypassing does not cause slow-down (except cactusADM) on least priority applications and provides substantial improvement on high and medium priority applications. Therefore, our assumption that 


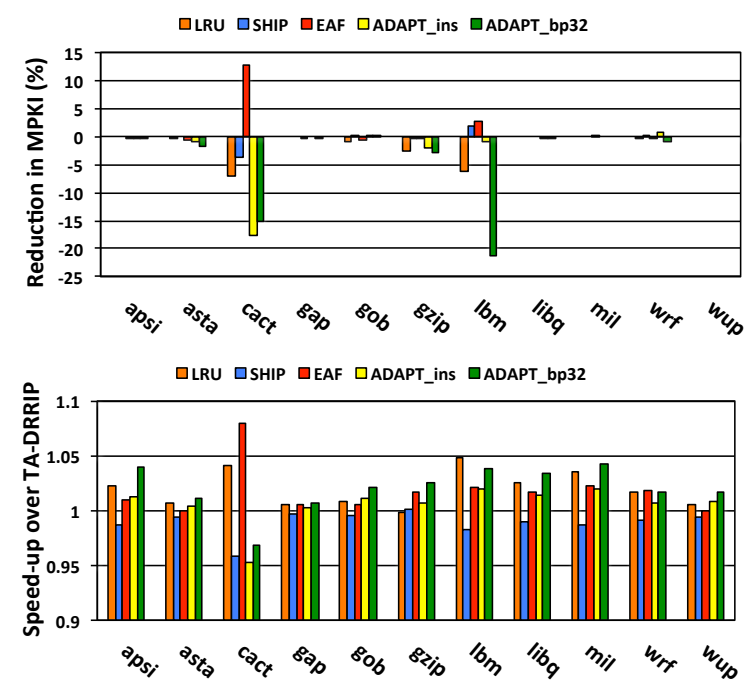

Figure 4: MPKI(top) and IPC(below) of thrashing

bypassing most of the cache lines of the least-priority applications to be beneficial to the overall performance is confirmed. As we bypass the cache lines (31/32 times) of the least-priority applications (instead of inserting), the cache state is not disturbed most of the times: cache lines which could benefit from staying in the cache remain in the cache longer without being removed by cache lines of the thrashing applications. For most of the applications bypassing provides substantial improvement in MPKI and IPC, as shown in Figure 5. Here, we report only applications with significant change $(>=3 \%)$ in MPKI or IPC. Bypassing affects only cactusADM. This is because some of their cache lines are reused immediately after insertion. Others do not show slow-down though their MPKI increases as with gzip and lbm. Because, an already memoryintensive application with high memory-related stall time, which when further delayed, does not experience much slow-down[20.

\subsection{Impact of Bypassing on cache replacement policies}

In this section, we show the impact of bypassing distant priority cache lines instead of inserting them on all replacement policies. Since LRU policy inserts all cache lines with MRU (high) priority, there is no opportunity to implement bypassing. From Figure 6, we observe that bypassing achieves higher performance for replacement policies except SHiP. As mentioned earlier, SHiP predicts distant reuse only for 3\% of the cache lines. Of them, $69 \%$ (on average) are miss-predictions. Hence, there is minor drop in performance.

On the contrary, TA-DRRIP, which implements bi-modal(BRRIP) on certain cache sets, bypasses the distant priority insertions directly to the private L2 cache, which is beneficial. Consequently, it learns BRRIP for the thrashing applications. Similarly, EAF with bypassing achieves higher performance. EAF, on average, inserts $93 \%$ of its cache lines with distant reuse prediction providing more opportunities to bypass. However, we observe 33\% (appx) of distant reuse predictions are incorrect7. Overall, from Figure 6, we can make two conclusions: first, our intuition of bypassing distant reuse cache lines can be applied to other replacement policies.

\footnotetext{
${ }^{7}$ Miss-predictions are accounted by tracking distant priority (RRPV 3 ) insertions which are not reused while staying in the cache, but referenced (within a window of 256 misses per set) after eviction. Here, we do not account distant priority insertions that are reused while staying in the cache because such miss-predictions do not cause penalty.
} 

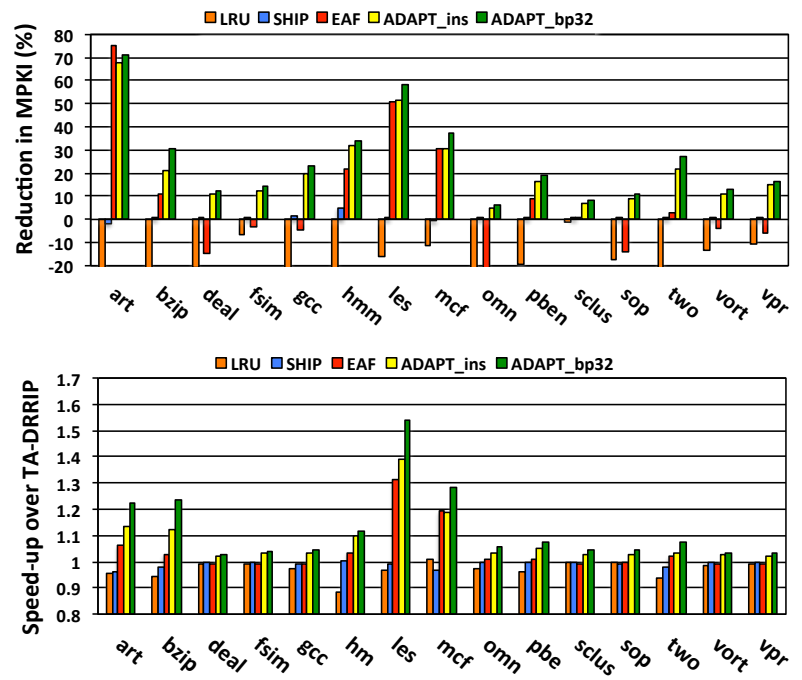

Figure 5: MPKI(top) and IPC(below) of non-thrashing

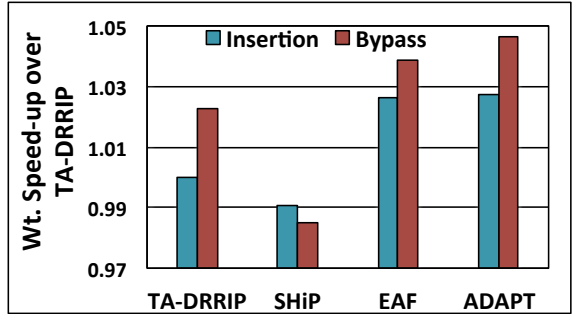

Figure 6: Impact of Bypassing on replacement policies

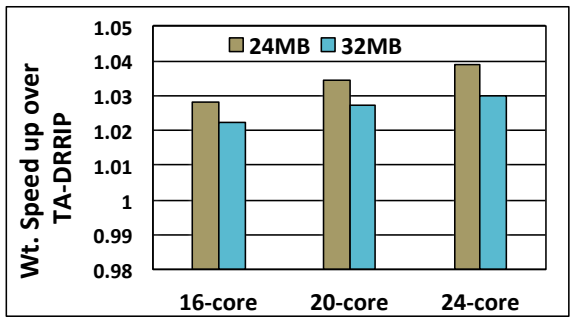

Figure 7: Performance on Larger Caches

Second, Footprint-number is a reliable metric to approximate an application's behavior: using Footprint-number, ADAPT distinguishes thrashing applications and bypasses their cache lines efficiently.

\subsection{Scalability with respect to number of applications}

In this section, we show the scalability of ADAPT with respect to the number of cores sharing the cache. Figure 8 shows the s-curves of the weighted speed-up for 4,8,20 and 24-core workloads. ADAPT outperforms prior cache replacement techniques. For 4-core workloads, ADAPT yields up to $20 \%$ improvement and $4.8 \%$ on average. For 8 -core workloads, ADAPT yields up to $9 \%$ improvement and 3.5\% approximately, on average. 20 and 24 -core workloads achieve $5.8 \%$ and 


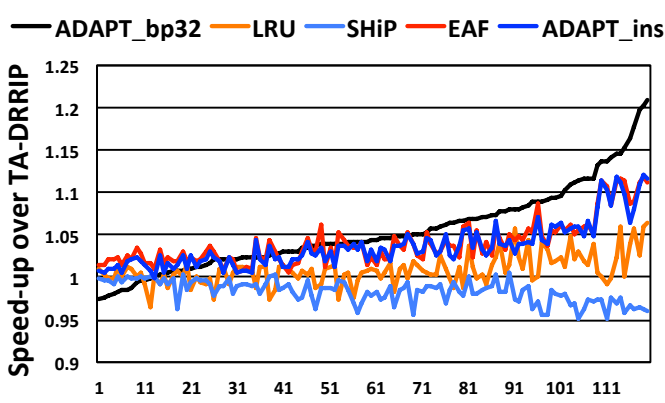

(a) 4-core (120 workloads)

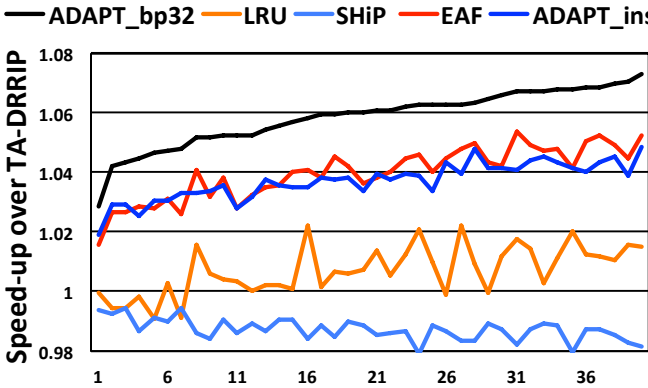

(c) 20 -core (40 workloads)

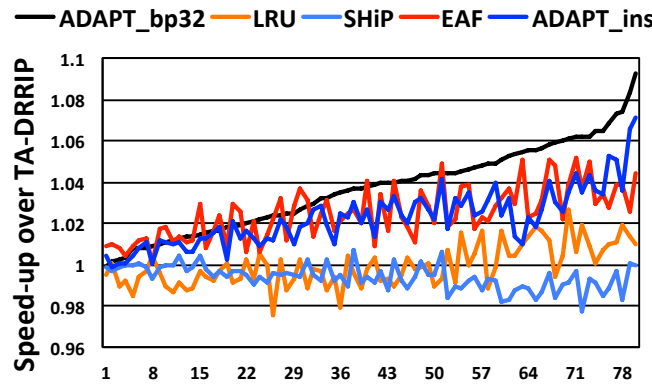

(b) 8-core (80 workloads)

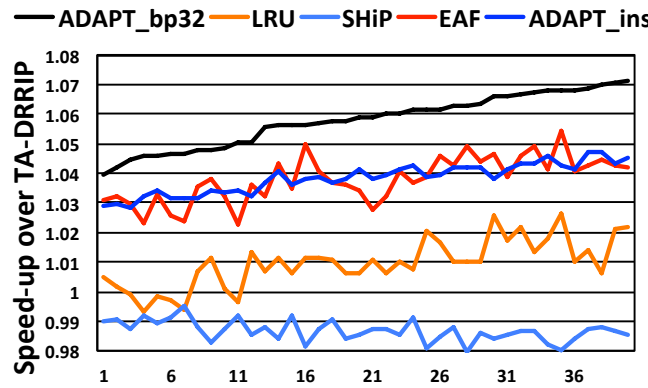

(d) 24-core (40 workloads)

Figure 8: Performance of ADAPT with respect to number of applications

$5.9 \%$ improvement, on average, respectively. Here, 20 and 24-core workloads are studied on 16MB,16-Way associative cache. Recall our proposition: (\#cores $\geq$ associativity).

\subsection{Sensitivity to Cache Configurations}

In this section, we study the impact of ADAPT replacement policy on systems with larger last level caches. In particular, the goal is to study if Footprint-number based priority assignment designed for 16-way associative caches applies to larger associative $(>16)$ caches as well. For $24 \mathrm{MB}$ and $32 \mathrm{MB}$ caches, we increase only the associativity of the cache set from 16 to 24 and 16 to 32 , respectively. Certain applications still exhibit thrashing behaviors even with larger cache sizes which ADAPT is able to manage and achieve higher performance on the weighted Speed-up metric(Figure 7).

\subsection{Other Multi-core Metrics}

Table 7 shows the performance of ADAPT on the Harmonic Mean of the Normalized IPCs, which balances both fairness and throughput [1] and the Harmonic, Geometric and Arithmetic Means of IPCs, which are consistent metrics for evaluation 27]. ADAPT shows similar improvement under these metrics as well. 
Table 7: Performance of ADAPT under other metrics

\begin{tabular}{|l|l|l|l|l|l|}
\hline Metric & 4-core & 8-core & 16-core & 20-core & 24-core \\
\hline Wt.Speed-up & $5.48 \%$ & $3.65 \%$ & $4.67 \%$ & $5.86 \%$ & $5.79 \%$ \\
\hline Norm. HM & $5.34 \%$ & $4.31 \%$ & $6.66 \%$ & $8.06 \%$ & $8.35 \%$ \\
\hline GM of IPCs & $5.43 \%$ & $3.98 \%$ & $5.34 \%$ & $6.8 \%$ & $6.95 \%$ \\
\hline HM of IPCs & $5.17 \%$ & $3.94 \%$ & $5.43 \%$ & $7.29 \%$ & $7.77 \%$ \\
\hline AM of IPCs & $5.27 \%$ & $3.27 \%$ & $4.82 \%$ & $5.85 \%$ & $5.63 \%$ \\
\hline
\end{tabular}

\section{Related Work}

Numerous studies have proposed novel ideas to manage multi-core shared caches. Here, we summarize some of them in the context of large-scale multi-core caches.

\subsection{Insertion priority prediction}

DIP [4] is one of the foremost proposals to alter the insertion priority of cache lines. In particular, they observe applications with working-set size larger than the cache to thrash under LRU and propose Bimodal policy for such workloads. DRRIP[1 predicts the reuse behavior of cache lines into re-reference interval buckets. RRIP consists of SRRIP and BRRIP policies. SRRIP handles mixed (recency-friendly pattern mixing with scan) and scan type of access patterns. BRRIP handles thrashing patterns. SHiP [5] and EAF [2] add further intelligence to the insertion predictions. SHiP uses Program-counter, Instruction sequence and Memory region signatures (separate mechanisms) to predict different priorities (SRRIP or BRRIP) for regions of accesses corresponding to the signature. EAF further enhances the prediction granularity to individual cache lines. A filter decides the SRRIP/BRRIP priority of cache lines based on its presence/absence in the filter.

All these approaches use only binary (SRRIP or BRRIP) insertion policies. Moreover, as discussed in the motivation section, they cannot be adapted to enable discrete prioritization. On the contrary, ADAPT is able to classify applications into discrete priority buckets and achieve higher performance. SHiP and EAF predict priorities at the granularity of (regions of) cache lines. However, in commercial designs [38, 40, which use SW-HW co-designed approach to resource management, the system software decides fairness or performance goals only at an application granularity. Hence, it is desirable that the cache management also performs application level performance optimizations.

\subsection{Reuse distance prediction}

Some studies [34, 36, 37, 35] compute the reuse distance values of cache lines at run-time to perform cache replacements. Since. Since the reuse distances of cache lines can take wider range of values, measuring reuse distance at run-time is typically complex, requires significant storage and modifying the cache tag arrays to store reuse distance values of cache lines. Schuff et al.[37] proposes a sampling and a parallel approach to measure the reuse distance of multithreaded applications. NUCache 32 propose a novel cache organization that builds on the idea of delinquent PCs. Cache is logically partitioned as main-ways and deli-ways.The idea is to store the cache lines (of delinquent PCs) evicted from the main-ways into deli-ways and retain the cache lines for duration beyond their eviction. The drawbacks with their approach is that caches need to have larger associativity, which adds significant energy overhead. Secondly, when there are large number of applications sharing the cache, finding the optimal set of delinquent PCs across all applications and assign deli-ways among them becomes complex.

\subsection{Eviction priority prediction}

Victim selection techniques try to predict cache lines that are either dead or very unlikely to be re-used soon [21, 22, 23, 25, 24]. A recent proposal, application-aware cache replacement [24] 
predicts cache lines with very long re-use distance using hit-gap counters. Hit-gap is defined as the number of accesses to a set between two hits to the same cache line. Precisely, the hit-gap gives the maximum duration for which the cache line should stay in the cache. On replacements, a cache line residing closer to/beyond this hit-gap value is evicted. In large multi-cores, under their approach, certain cache-friendly applications could get hidden behind the memory-intensive ones and suffer more misses. However, ADAPT would be able to classify such applications and retain their cache lines for longer time. Further, this mechanism requires expensive look-up operations and significant modifications to the cache tag array.

\subsection{Cache Bypassing}

Many studies have proposed bypassing of cache lines [13, 14, 18, 17, 12]. All these techniques either completely bypass or insert all requests. For thrashing applications, retaining a fraction of the working set is beneficial to the application 44. However, in larger multi-cores, such an approach is not completely beneficial. Inserting cache lines of thrashing applications with leastpriority still pollutes the cache. Instead, bypassing most of their cache lines is beneficial both to the thrashing application as well as the overall performance. As we show in Section 5.3. bypassing least-priority cache lines is beneficial to other replacement policies as well. Segmented-LRU 12 learns the benefit of bypassing on randomly selected cache lines by observing the relative timing of the victim/non-bypassed or retained/bypassed cache lines. However, observing the hits/misses on the shared cache is not an efficient way to decide on policies as they may lead to inefficient decisions. Gaur et al.[16] propose bypass algorithm for exclusive LLCs. While they study bypassing of cache blocks based on its L2 use and L2-LLC trip counts, our bypass decisions are based on the working-set size of applications. [15] uses data locality to manage placement of cache lines between Private L1 and Shared L2. Only high locality lines are inserted at L1 while cache lines with low locality are not allocated. The principal difference from our approach is that they manage private caches by forcing exclusivity on select data while we manage shared caches by forcing exclusivity on select application cache lines.

\subsection{Cache partitioning techniques}

Cache partitioning techniques $7,8,6,6,10$, focus on allocating fixed-number of ways per set to competing applications. Typically, a shadow tag structure (that exploits stack property of LRU [26] [6] monitors the application's cache utility by using counters to record the number of hits each recency-position in the LRU stack receives. Essentially, the counter value indicates the number of misses saved if that cache way were allocated to that application. The allocation policy assigns cache ways to applications based on their relative margin of benefit. While these studies suffer from scalability with number of cores, some studies have proposed novel approaches to fine-grained cache partitioning [30, 29, 31] that break the partitioning-associativity barrier. These mechanism achieve fine-grained (at cache block level) through adjusting the eviction priorities. Jigsaw [30 leverages Vantage 31] for the cache hardware, but uses a novel software cache allocation policy based on the insight that miss-curves are typically non-convex and this property provides scope for efficient and a faster allocation algorithm. PriSM [29] proposes a pool of allocation policies which are based on the miss-rates and cache occupancies of individual applications. Essentially, these mechanisms require quite larger associative caches. For tracking per-application utility, 256-way LRU managed shadow tags are required [30] 31]. Further, these techniques require significant modification to the existing cache replacement to adapt to their needs. Contrastingly, ADAPT does not require modifying the cache states. Only the insertion policies are altered. 


\section{Summary and Future Work}

Future multi-core processors will continue to employ shared last level caches. Sharing caches in large multi-cores poses two new challenges: (i) the ability to manage (\#cores $\geq$ \#associativity) and (ii) the replacement policy must be application aware and allow discrete $(>2)$ prioritization of applications. These challenges require an efficient and a scalable cache monitoring mechanism that allows the replacement policy in meeting these above goals. Towards this end, we propose a new cache monitoring mechanism and an insertion-priority-alogirhtm. In summary, we make the following contributions:

-We identify that existing approach of observing hit/miss pattern to approximate applications' behavior is not efficient. Such an approach causes run-time cache replacement decisions to be suboptimal.

-We introduce the Footprint-number metric to dynamically track the working-set size of applications. We propose Adaptive, Discrete and de-prioritized Application PrioriTization (ADAPT), which consists of a monitoring mechanism and an insertion-priority-prediction algorithm. The monitoring mechanism dynamically captures the Footprint-number of applications on an interval basis. The prediction algorithm computes insertion priorities for applications from the Footprintnumbers under the assumption that smaller the Footprint-number, better the cache utilization. From experiments we show ADAPT is efficient and scalable ((\#cores $\geq$ \#associativity $))$.

Though our study is performed with L1 cache prefetcher, commercial processors typically employ mid-level cache(L2) prefetching. We intend to study large multi-core shared caches with L2 prefetching in the future.

\section{References}

[1] Aamer Jaleel, Kevin B. Theobald, Simon C. Steely, Jr., and Joel Emer. High performance cache replacement using re-reference interval prediction (RRIP). In ISCA 2010.

[2] Vivek Seshadri, Onur Mutlu, Michael A. Kozuch, and Todd C. Mowry. The evicted-address filter: a unified mechanism to address both cache pollution and thrashing. In PACT 2012.

[3] Aamer Jaleel, William Hasenplaugh, Moinuddin Qureshi, Julien Sebot, Simon Steely, Jr., and Joel Emer. Adaptive insertion policies for managing shared caches. In PACT 2008.

[4] Moinuddin K. Qureshi, Aamer Jaleel, Yale N. Patt, Simon C. Steely, and Joel Emer. Adaptive insertion policies for high performance caching. In ISCA 2007.

[5] Carole-Jean Wu, Aamer Jaleel, Will Hasenplaugh, Margaret Martonosi, Simon C. Steely, Jr., and Joel Emer. SHiP: signature-based hit predictor for high performance caching. In MICRO 2011.

[6] Moinuddin K. Qureshi and Yale N. Patt. Utility-Based Cache Partitioning: A LowOverhead, High-Performance, Runtime Mechanism to Partition Shared Caches. In MICRO 2006.

[7] Kyle J. Nesbit, James Laudon, and James E. Smith. Virtual private caches. In ISCA 2007.

[8] Yuejian Xie and Gabriel H. Loh. PIPP: promotion/insertion pseudo-partitioning of multicore shared caches. In ISCA 2009.

[9] Ravi Iyer. 2004. CQoS: a framework for enabling QoS in shared caches of CMP platforms. In ICS 2004. 
[10] Gupta, A.; Sampson, J.; Taylor, M.B., TimeCube: A manycore embedded processor with interference-agnostic progress tracking, In SAMOS 2013.

[11] Mainak Chaudhuri, Jayesh Gaur, Nithiyanandan Bashyam, Sreenivas Subramoney, and Joseph Nuzman. Introducing hierarchy-awareness in replacement and bypass algorithms for last-level caches. In PACT 2012.

[12] Hongliang Gao, Chris Wilkerson. A Dueling Segmented LRU Replacement Algorithm with Adaptive Bypassing. Joel Emer. JWAC 2010 - 1st JILP Worshop on Computer Architecture Competitions: cache replacement Championship.

[13] Jamison D. Collins and Dean M. Tullsen. Hardware identification of cache conflict misses. In MICRO 1999.

[14] Teresa L. Johnson, Daniel A. Connors, Matthew C. Merten, and Wen-mei W. Hwu. RunTime Cache Bypassing. in IEEE TC, 1999.

[15] George Kurian, Omer Khan, and Srinivas Devadas. The locality-aware adaptive cache coherence protocol. In ISCA 2013.

[16] Jayesh Gaur, Mainak Chaudhuri, and Sreenivas Subramoney. Bypass and insertion algorithms for exclusive last-level caches. In ISCA 2011.

[17] Antonio González, Carlos Aliagas, and Mateo Valero. A data cache with multiple caching strategies tuned to different types of locality. In ICS 1995.

[18] Scott McFarling. 1992. Cache replacement with dynamic exclusion. In ISCA 1992.

[19] Velasquez, R. A and Michaud, P. and Seznec, A. BADCO: Behavioral ApplicationDependent Superscalar Core model. In SAMOS 2012.

[20] Onur Mutlu and Thomas Moscibroda.Parallelism-Aware Batch Scheduling: Enhancing both Performance and Fairness of Shared DRAM Systems. In ISCA 2008.

[21] Wei-Fen Lin and Steven K. Reinhardt,2002, Predicting Last-Touch References under Optimal Replacement

[22] Haiming Liu, Michael Ferdman, Jaehyuk Huh, and Doug Burger. Cache bursts: A new approach for eliminating dead blocks and increasing cache efficiency. In MICRO 2008

[23] An-Chow Lai, Cem Fide, and Babak Falsafi. Dead-block prediction \& dead-block correlating prefetchers. In ISCA 2001.

[24] Tripti S. Warrier, B. Anupama, and Madhu Mutyam. An application-aware cache replacement policy for last-level caches. In ARCS 2013.

[25] Samira Manabi Khan, Yingying Tian, and Daniel A. Jimenez. 2010. Sampling Dead Block Prediction for Last-Level Caches. In MICRO 2010.

[26] R. L. Mattson, J. Gecsei, D. R. Slutz, and I. L. Traiger. Evaluation techniques for storage hierarchies. IBM Syst. J. 9, 2 (June 1970),

[27] Pierre Michaud. Demystifying multicore throughput metrics. IEEE Comp. Arch. Letters, June 2013.

[28] Zhao Zhang, Zhichun Zhu, and Xiaodong Zhang. A permutation-based page interleaving scheme to reduce row-buffer conflicts and exploit data locality. In MICRO 2000. 
[29] R Manikantan, Kaushik Rajan, and R Govindarajan. Probabilistic shared cache management (PriSM). In ISCA 2012.

[30] Daniel Sanchez and Christos Kozyrakis. Vantage: scalable and efficient fine-grain cache partitioning. In ISCA 2011.

[31] Nathan Beckmann and Daniel Sanchez. Jigsaw: scalable software-defined caches. In PACT 2013.

[32] R Manikantan, Kaushik Rajan, and R Govindarajan. NUcache: An efficient multicore cache organization based on Next-Use distance. In HPCA 2011.

[33] Keramidas, G.; Petoumenos, P.; Kaxiras, S., "Cache replacement based on reuse-distance prediction," in ICCD 2007.

[34] Mazen Kharbutli, Yan Solihin, "Counter-Based Cache Replacement and Bypassing Algorithms", IEEE Transactions on Computers, April 2008.

[35] Nam Duong, Dali Zhao, Taesu Kim, Rosario Cammarota, Mateo Valero, and Alexander V. Veidenbaum. In MICRO 2012.

[36] David Eklov, David Black-Schaffer, and Erik Hagersten. Fast modeling of shared caches in multicore systems. In HiPEAC 2011.

[37] Derek L. Schuff, Milind Kulkarni, and Vijay S. Pai.Accelerating multicore reuse distance analysis with sampling and parallelization. In PACT 2010.

[38] https://software.intel.com/en-us/blogs/2014/06/18/benefit-of-cache-monitoring

[39] http://blog.stuffedcow.net/2013/01/ivb-cache-replacement/

[40] http://www.intel.com/content/www/us/en/architecture-and-technology/64-ia-32architectures-software-developer-vol-3b-part-2-manual.html

[41] Kun Luo; Gummaraju, J.; Franklin, M.,"Balancing thoughput and fairness in SMT processors," in ISPASS 2001. 


\section{Contents}

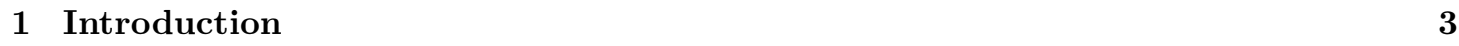

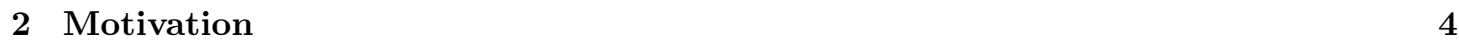

3 Adaptive discretized and de-prioritized cache insertions (ADAPT) 5

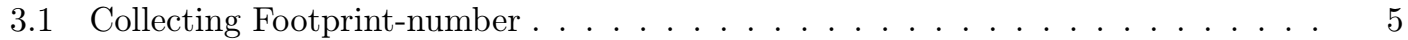

3.2 Footprint-number based Priority assignment . . . . . . . . . . . . . . . . . . . . . 7

3.3 Hardware Overhead $\ldots \ldots \ldots \ldots$. . . . . . . . . . . . . . . . . . . . . 8

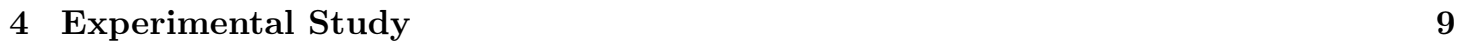

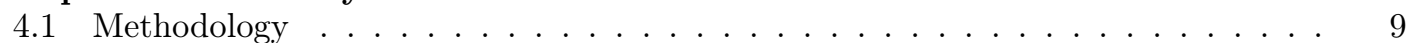

4.2 Benchmarks . . . . . . . . . . . . . . . . . . . . . . . . . . . . . . . . . . . . . . . . 9

4.3 Workload Design . . . . . . . . . . . . . . . . . . . . . . . . 10

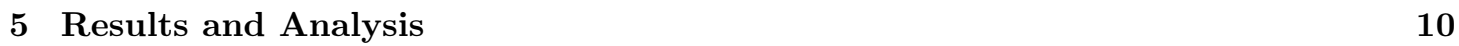

5.1 Performance on 16-core workloads . . . . . . . . . . . . . . . . . . . . 10

5.2 Impact on Individual Application Performance . . . . . . . . . . . . . . . . . . . 11

5.3 Impact of Bypassing on cache replacement policies . . . . . . . . . . . . . . . . . 12

5.4 Scalability with respect to number of applications . . . . . . . . . . . . . . . . . . 13

5.5 Sensitivity to Cache Configurations . . . . . . . . . . . . . . . . . . . . . . 14

5.6 Other Multi-core Metrics _ . . . . . . . . . . . . . . . . . . . . . . . . . . 14

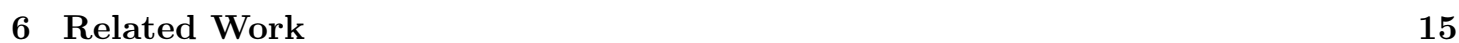

6.1 Insertion priority prediction . . . . . . . . . . . . . . . . . . 15

6.2 Reuse distance prediction . . . . . . . . . . . . . . . . . . . . . . . . . . 15

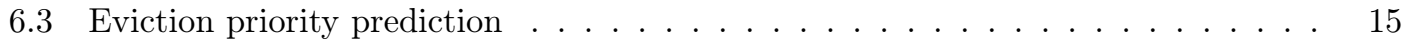

6.4 Cache Bypassing . . . . . . . . . . . . . . . . . . . . . . 16

6.5 Cache partitioning techniques . . . . . . . . . . . . . . . . . . . . . . . 16

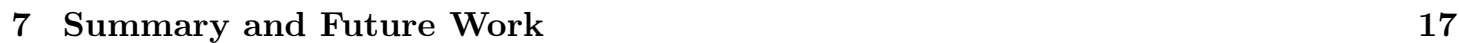




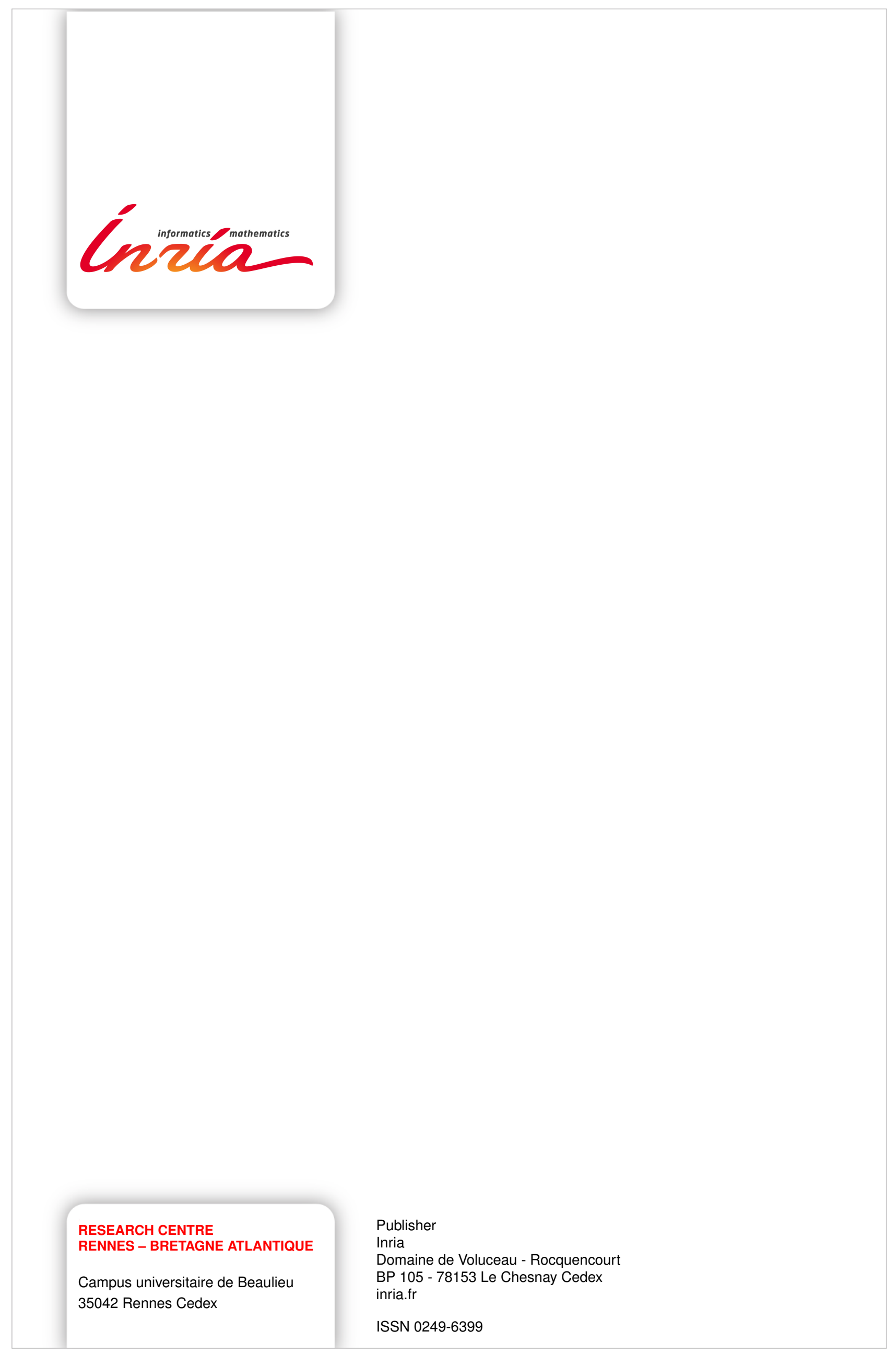

